\title{
The Human Body-Field
}

\author{
Edward F Block* \\ Acupuncturist, Block Institute for Astrobiological Studies, USA
}

*Corresponding author: Edward F Block, Acupuncturist, Block Institute for Astrobiological Studies, 160 Holiday Loop, Unit 2, New Braunfels, TX 78132.
Received Date: June 04, 2020

Published Date: June 22, 2020

\section{Introduction}

All holistic healing modalities purport to make the human body an integrated dynamic system of matter and energy. The human body is in harmony within itself and within the overall environment that it inhabits. Any disharmony within the body then is the basis of what is known as dis-ease. The dis-harmonic portion of overall integrated body-field eventually results in the disruption the rheostatic processes within the body. The physical body will then display symptoms of dis-ease according to that part of the overall integrated body-field that is in dis-harmony. This Dissertation will endeavour to elucidate the characteristics of the Human BodyField and then the role that holistic healing modalities play in the maintenance of an integrated and optimally functioning human body.

\section{Discussion}

\section{The basic physics of earth science}

This portion is not meant to be definitive but instead of as early giving a basic grounding in current thought in the field of Physics and Earth Science. The realities of the universe have shown that there are four fundamental forces (or rather fundamental interactions) that are the ways that individual particles of matter interact with each other. It turns out that every single interaction observed taking place in the universe can be broken down and described by only four types of interactions: Gravity, Electromagnetism, Weak Interaction (or Weak Nuclear Force) and Strong Interaction (or Strong Nuclear Force). Only gravity and electromagnetism is pertinent to this discussion. The force of gravity means that matter tends to collect together. The collections may be of mostly hydrogen, the most abundent substance, and yield densities that produce thermonuclear interactions that result in what are known as Stars. The collections may be made up of left over materials from dead stars and yield densities that produce planets.

The interactions of matter are such as to produce the most stable atomic and molecular conditions. These interactions are mediated by what is known as light or the electromagnetic spectrum. As the wavicles of light packets traverse space, it produces along a spiral path two attendent fields, each perpendicular to each other. The electric field is along the path of traverse and the magnetic field extends perpendicular to it. The electromagnetic spectrum is how man describes the amazing display of light from very energetic short wavelength high frequency wavicles to quite low energy long wavelength low frequency wavicles.

The parameters of the Solar System that our Sun and attendent planets, asteroids, comets and such makes up are made of dynamic interactions with the Sun at the center, all due to gravity. The Planet we call Earth is the 3rd from the sun and as such is in a position that is suitable for life to emerge and evolve to yield what is evident today. What is important for this discussion is the direct interaction of the Solar radiations from the Sun as they impact the Earth.

The Earth is made up of all manner of different elements that have combined into the molecules that make up the substances of the Earth. A principle of physics shows us that the heaviest substances are at the core of the planets and the lighter more to the surface. The core of the planet Earth is mostly Iron. A very significant fact is that this molten core is spinning with the surface spinning in time with a lag due to friction. The spinning core produces a magnetic field that radiates into space and is called the magnetosphere. Thus, the planet Earth acts like a giant magnet. 
The radiations from the Sun impact the Earth. These radiations are composed of light, electrons and protons. The magnetic field protects the surface of the Earth from the harmful to life radiations of electrons and protons. The light component reaches the surface of the planet. As the planet spins upon its axis, this light interacts with surface matter and warms it. The planet Earth is tilted 23 $1 / 2$ degrees from verticle and the distribution of light across the surface and in the atmosphere produces the weather patterns that distribute wind, wind blown matter and water as rain or snow across the surface.

The Biosphere is that surface area of the planet within which life exists, by definition. The Biosphere is made up of three components: atmosphere, hydrosphere, lithosphere. Life has evolved within the biosphere but without the hydrosphere, composed of water and mineral substances dissolved within the water, life would not have been able to exist.

Two phenomena are constantly taking place within the Biosphere that are pertinent to this discussion from the standpoint of Physics and Earth Science that greatly influence the human condition. One is the generation of what are called the Schumann Resonances and the other is the generation of what are known as the Giertz Frequencies.

In 1952, WO Schumman [1] described events that occur in the ionisphere that produce a planet wide resonant frequency of $7.83 \mathrm{~Hz}$. At any given moment about 2,000 thunderstorms roll over the Earth, producing some 50 flashes of lightning every second. Electrons are discharged into the resonant cavity formed by the surface of the planet and the ionisphere. The fundamental frequency of the resonance is $7.83 \mathrm{~Hz}$ (along with progressively weaker harmonics at 14.3, 20.8, 27.3 and $33.8 \mathrm{~Hz}$.) which is the same as the alpha rhythm of mammalian brain activity.

In 2010, HW Giertz [2] published an equation that gave insight as to the frequencies of matter and their relation to life phenomena. In particular, the frequency of $4.50 \mathrm{~Hz}$ which coincides with the resonant frequency of water.

\section{The discoveries of the meditational arts}

The author has been meditating since the age of 4 years and completely self taught. The information provided is the result of over 70 years of meditation practice. The practice of meditation is very right-brain in expression. The author is a very well trained scientist and has delved into the realm of left-brain experience. The result is a combination of experience that is wholistic in outlook.

The origins of meditation are unknown and are a completely natural outgrowth in the development of consciousness. The first written works dealing with meditation are from the sub-continent of India and the terms utilized here will be from Raja Yoga. However, it is from the personal experience of the author that discussion will be offered.
To the Westerner, the Staff of Hermes (the Caduceus) is the representation of the energetic field which subtends the physical body. The staff itself is the Sushumna and the 2 snakes that entwine the staff are the Ida and the Pingala. The points of crossover (the nodes) from side to side are the Chakras of which there are 7 (Basal $\{-3\}$, Sacral $\{-2\}$, Abdominal $\{-1\}$, Heart $\{0\}$, Throat $\{+1\}$, Brow $\{+2\}$, Crown $\{+3\}$ ). The inter-nodes are represented by the visible light frequencies which coincide with the 7 electromagnetic spectrum colors of Red, Orange, Yellow, Green, Blue, Indigo, Violet and 1 of gold. The Red color is very much tied to the negative energies of the planet and is a dual truncated cone pointing downward from the area of the basal chakra. The Gold color is a dual truncated cone pointing upwards from the top of the head and very much tied to the energies of the Solar System. The other colors are represented by flat discs or spinning wheels between the nodes. The Ida and Pingala energies are Clock-wise and Counter Clock-wise in spin orientation and are 2 resonant sinosoidal frequencies generated by the Chakra matrix as the Sushumna and nodes represented by the 2 snakes entwined about the Staff.

As stated, the basal node is the crossover point for the entrance of negative energies and frequencies $(4.50 \mathrm{~Hz})$ from the planet to enter the chakra matrix just as the positive energies and frequencies $(7.83 \mathrm{~Hz})$ from the Solar System enter the matrix at the Crown node and the harmonics of these standing waves according to the chakra matrix.

The orientation of the Chakral matrix is perpendicular to the core of the Earth. The negative energies enter the physical body from the front at the feet and move up the front of the body and into the body at the acupuncture point Ren 1 each subsequent node producing a rotating cylinder with CW spin on the right and CCW spin on the left all the way up to acupuncture point Du20 . The positive energies move from Du20 down the backside of the body and contribute to the spin at each subsequent node ending at Du1. This sets the stage for the 7 inter-nodes as resonant cylinders with $(+, \mathrm{CW})$ and (-, CCW) fields and spin according to and the interaction with the environment that the whole organism resides within the Biosphere. The entire overall field matrix is optimally balanced with the free flow of resonant and harmonic frequecies according the the genetic makeup of the physical body.

The human body nervous system is generally divided into 2 portions. The Central Nervous System (CNS) is the brain and the spinal cord. The Peripheral Nervous System (PNS) is the gut plexi and sensory input. Roughly the same nunber of nervous elements are in each of the 2 with the vagus nerve as the direct connection between the 2 portions. The PNS is involved with sensory input and the gathering of nutrient substances that go to make up the physical body. The CNS is involved with conscious volition, autonomic coordination and hormonal responses to the fluctuations in the environment. The frontal portion of the brain determines voluntary 
skeletal muscle activation. The hindbrain is tied to visual input and processing. The pre-frontal areas of the brain determine intent (Brow Node).

\section{The Human Body-Field}

The overall human body-field (Aura)is toroidal in shape just like the overall Geomagnetic field of the Earth. However, with conscious intent, it is possible to create 2 overlapping fields represented as pyramids. These bases of these pyramids may then be moved from the center ( 0 ) node and extended to the Basal and Crown chakras. This creates a box. This "Box" is the body temple, as it were. The normal everyday state of the Aura is the toroidal shape. The "Box" is a state for optimal healing, more upon this topic later.

At each of the inter-nodes there are 2 orientations: front to back (Ren, neutral and $\mathrm{Du}$ ) and left (-), neutral and right (+). Thus there are 21 ( 7 times 3 states) different discrete areas within the overall horizontal aspect of the Aura, apart from the Ren and Du. The 6 "wheels" are arranged in 12 left and right side circular segments from the midline measured as back to front on each side from 0 degrees to 180 degrees. The direction of influence is through your body, back to front in direction, for a total of 12 wheel segments on each side. Twelve CW (-) segments stepwise from the midline at the back and 12 CCW (+) segments stepwise to the opposite side from the back, in 15 degree arcs. These are the 12 sub-sets of the 2 left-side and right-side main horizontal components. Thus, each segment of the spiral turn is in a 15 degree arc of influence with a time component determined by the combination of the transverse component and the incremental side component. Each of the 12 (+) and (-) 15 degree arc wheel segments takes a total of 2 hours for transit, according to traditional Chinese medicine (TCM). Each wheel transit therefore takes 24 hours to complete. Thus all 7 anti-nodes are transiting at the same time in synchrony, both in the (+) and (-) sets of the left and right spiral components. Being healthy means that the (+) and (-) spiral flows are synchronous and balanced. As the wave becomes the opposite sign at the next node, this means that the 12 sub-sets need to be balanced from left to right and back again. This also means that you are whole and in good health. The 8 dual $(+)$ and $(-)$ wheels or anti-nodes of the spiral wave are the regulators of the energy systems of the body. The 12 wheel segments of each wheel are the integrators and regulators of the already in place physiology of the body. The various compartments of the tissues and organs of the body, but especially the cells that make up the tissues and organs of the body are integrated into a metabolic whole by this system.

\section{The role of holistic healing modalities}

The real pioneer in the field of natural healing in modern times is Franz Friedrich Anton Mesmer \{1734-1815\} [3]. That he did not understand what he was working with is evident in the methods of his practice and the works written about his healing modality. His methods did effect "cures" in the body and mind of his patients. This fact leads one to understand that Intent (Brow node) is everything in the Art of Healing. The portion of the Aura that is in imbalance (1 of the 21 areas or multiples thereof) will need to be returned to balance for a "cure" to occur.

Perhaps it is of a time to speak about what improves balance of the Aura and what seems to cause an imbalance. A change in the Aura will always proceed that of a change in physiology. This is why the application of pharmaceutical drugs to influence physiology do not actually cure but in fact poison the body. If you use an instrument that will detect Extemely Low Frequencies (ELF), areas within the physical body that are infected with pathogenic bacteria/fungi will resonate at frequencies just above and below that of the frequency of pure, not contaminated water at $4.50 \mathrm{~Hz}$, as stated above. These 2 frequencies are at a (+,-) $0.75 \mathrm{~Hz}$ difference at $3.75 \mathrm{~Hz}$ and $5.25 \mathrm{~Hz}$. Dr. Giertz also found that the human condition of cancer resonated at $3.00 \mathrm{~Hz}$. Harmful pharmaceutical drugs resonated at ELF frequencies at mutliples of $0.75 \mathrm{~Hz}$ above that of pure water $(4.50 \mathrm{~Hz})$.

According to Dr. Giertz, the Birkeland currents [4] of electrons traveling in the crust of the Earth interact with the Geomagnetic field produced by the dynamic spinning core of the Earth to then result in transverse electromagnetic waves in the ELF region perpendicluar the the surface of the Earth. The strongest is that of $4.5 \mathrm{~Hz}$ with harmonics of decreasing strength always at multiples of $0.75 \mathrm{~Hz}$. This in the causal effect that has also been previously named the Hartmann and Curry Grids by these 2 Medical Doctors in the field of geopathic stress as it effects human health.

Ernst Hartmann described this planetary Grid in his book entitled "Krankheit als Standortproblem (Illness as a Location Problem)", 1954 [5]. Manfred Curry wrote about his Grid in 1952 that was actually discovered by Siegfried Wittmann in 1950. I have not been able to verify online this information about the Curry Grid. Searches yield many sites that allude to the Geopathic Stress the these 2 Grids cause in people sleeping on portions of the Grid.

One of the Sayings of Hippocrates is to "Let food be thy medicine and medicine be thy food". And assuredly this is all too true. However, in this day and times it is difficult to find food that is not in some manner contaminated by the various food producing entities. The main reason that food crops were genetically modified was for them to not be effected by the herbacide Roundup (Glyphosate), specifically for crops of soybeans, corn and canola (Rapeseed).

Glyphosate is a broad-spectrum systemic herbicide and crop desiccant. It is an organophosphorus compound, specifically a phosphonate, which acts by inhibiting the plant enzyme 5-enolpyruvylshikimate-3-phosphate synthase. It is used to kill weeds, especially annual broadleaf weeds and grasses that 
compete with agracultural crops. Monsanto brought it to market for agricultural use in 1974 under the trade name Roundup. Glyphosate is now known to be a cause of cancer in mammals [6].

Another major culprit in the cause of obesity, diabetes and arthritis is Soybean oil. This is due to the trace amounts of herbacides, pesticides and natural phytoestrogens contained in the oil. Today, soybean oil is found in almsot all processed food products. The herbacides and pesticides disrupt cellular metabolism within the body and the phytoestrogens artificially stimulate cartilage production in the joints causing arthritis.

Another major cause of aura disharmony is electromagnetic pollution, especially that at the frequecies of and the harmonics of the Giertz frequencies and the Schumann resonances. The work of scientists have now shown that the result of overall physiology in the brain is more of a magnetic than electrical phenomena [79]. Insurance actuaries have known since the 1920s that people exposed daily to the effects of motors live shorter lives and get ill more often than others [10,11].

So how does one maintain the aura at its optimum for continued good health? Other than eating well, keeping out of geopathic zones and away from electromagnetic pollution there are healing modalities that directly address the issues of aura imbalance?

The premire healing modality is Homeopathic medicine followed by Naturopathy and Acupuncture/Oriental medicine and massage. Very much information about these modalities may be found online. Both Homeopathy and Oriental medicine utilize plant and animal substances to harmonize the Aura. Naturopathy, Acupuncture and massage act directly upon the body-field.

I did not mention Reiki [12] as a healing modality as it is just another name for Mesmerism like the so called Orgone therapy [13], in the opinion of the author. A secret is being revealed in the following statement - any healing that involves the close proximity between patient and healer involves the transfer of energies from the aura of the healer to the patient and vice versa. This 2-way transfer is the cause of "Burn Out" in healthcare providers. This fact is also the cause of what I know as psychic vampirism by those not able to take care of themselves and are constantly draining the lifeforce from those around them.

\section{Healing protocol}

What follows is the personal method by the author of effecting healing in another person.

I have not yet found any suitable information concerning this topic online and thus I will describe the protocol that I use when treating a patient. My body-field is the instrument that I use to check the patient body-field and the pendulum that I use is the instrument that I use to check my sensitivity (dowsing). After the 30 minutes of acupuncture treatment, I will check the efficacy of the treatment and determine if there is any detectable body-field disharmony by dowsing with my pendulum. I make sure that I am well grounded within myself to the core of the Earth and centered in my body-field before I attempt to dowse. This will mean that I have sufficient experience and the techniques to accomplish this feat of total centered and grounded beingness. I then take my pendulum and start a scan with my personally determined neutral swing, in my case a to and for swing from the center of my body. The patient is lying on their back with the arms not touching the body and the hands located to allow me to scan the whole arm. I start at the sole of the foot (either) and scan up the leg to the hip. Next I scan the other leg from sole to hip. Then I scan the trunk of the body up the mid-line from the crotch to the chin. I them move to the hip and scan from hip (either) to shoulder and then repeat on the other side. Next I scan in turn both arms from fingers to shoulder. Last is a scan of the head; from chin to the top and then from chin along the jaw to the ear and the side of the head. I repeat the scan for the other side of the head to complete the sequence.

If the patient has no disharmony, I will observe my neutral swing throughout the entire procedure. I scan slowly so that any disharmony will be evident at any particular location. The pendulum will begin to rotate, to the left or to the right, if my bodyfield will detect a disharmony in that of the patient. It matters not the spin orientation but I with intent counter the patient's swing by purposely rotating my pendulum in the opposite direction with an intuitively determined number of counter swings. At the time that I feel that a sufficient number of counter rotations has occurred, I remove the pendulum from the site to a distance away from the patient and allow my swing to become neutral again. I then approach the patient and scan the site of disharmony to test the efficacy of my treatment at that spot on the patient's body. If I am tuned in, there will be no rotation in the same direction as before but will remain neutral. If I have errored on the side of too little the same rotation will occur but in a diminished mode. If I have overcompensated, the rotation will be reversed. I adjust as needed to bring about the neutral swing with my pendulum. Throughout the entire process, I must remain grounded and centered. I complete the scan in entirety until no rotations are detected. This completes the protocol.

During the time that I am countering any spin that I detect in a body location from a patient, I visualize (with intent) my bodyfield energies creating an opposite spin to that encountered. The intensity of my projection needs to match the intensity of the spin detected in the patient. Also, the number of counter turns needs to match the intensity encountered. With practice, right-brain intuitive processes will tune you to this protocol and you will be able to smoothly complete the protocol in an appropriate manner practice makes perfect!

\section{Conclusion}

The matter and the attendent energies that make up the physical human body are a complex of interacting fields of 
influence labeled as the morphogenic field. This field is the result of the genetic processes that started upon fertilization of the oocyte released by the ovum of the mother body. The gestational period of 270 days results in a new human being. This new human being displays overlapping fields of energetic influence characterized by an integrated overall human body field. This "aura" field is the result of evolutionary processes and have been passed down to posterity via genetic processes. These genetic processes have developed within the biosphere of the Earth. The characteristics of Earth processes are the backdrop within which these genetic processes have developed. The human body then somewhat mimics certain aspects of the Earth and its processes but also being amended for being a living being. Holistic healing directly addresses the processes within the human body-field in order to assist people in maintaining optimum health in physical body and mind.

\section{Acknowledgement}

None.

\section{Conflict of Interest}

The authors declare no competing interest.

\section{References}

1. Schumann WO (1952) Über die Ausbreitung sehr Langer elektriseher Wellen um die Signale des Blitzes. Nuovo Cimento 9(12): 1116-1138.
2. Giertz HW (2010) Extremely low frequency electromagnetic energy in the air. Journal of Atmospheric and Solar-Terrestrial Physics 72(9-10): 767-773.

3. Franz Mesmer.

4. Birkeland current.

5. Ernst Hartmann (1954) Krankheit als Standortproblem (Illness as a Location Problem).

6. Guyton KZ, Loomis D, Grosse Y, El Ghissassi F, Tallaa LB, et al (2015) Carcinogenicity of Tetrachlorvinphos, Parathion, Malathion, Diazinon, and Glyphosate. Lancet Oncol 16(5): 490-491.

7. Clay Dillow (2010) A Magnetic Field Applied to the Brain Can Alter People's Sense of Morality.

8. Erica Tennenhouse (2018) What Magnetic Fields Do to Your Brain and Body.

9. Electromagnetic theories of consciousness.

10. (1992) Electromagnetic Fields and Circadian Rhythmicity. In: MC Moore Ede, SS Campbell RJ Reiter (eds), Birkhouser Boston, USA.

11.E Sobel, M Dunn, Z Davanipour, Z Qian, HC Chui (1996) Elevated Risk of Alzheimer's Disease Among Workers With Likely Electromagnetic Field Exposure. Neurology 47(6): 1477-1481.

12. Reiki.

13. Orgone. 